\title{
Overview and highlights of the Spin Asymmetries of the Nucleon Experiment - SANE
}

\author{
Oscar A. Rondon ${ }^{\mathrm{a}}$ \\ Institute for Nuclear and Particle Physics, University of Virginia, Charlottesville, VA 22903, USA
}

\begin{abstract}
The Spin Asymmetries of the Nucleon Experiment - SANE took data on inelastic and elastic inclusive and coincidence beam-target asymmetries in the Hall $\mathrm{C}$ facility at Jefferson Lab, detecting the scattered electrons with the large non-magnetic Big Electron Telescope Array - BETA and the High Momentum Spectrometer. The Continuous Electron Beam Accelerator Facility - CEBAF delivered polarized electrons that were incident on the U. of Virginia polarized proton target. An overview and selected examples of the wealth of collected data, extending from the elastic to the deep inelastic scattering regions, and spanning the four-momentum range from 0.8 to $6 \mathrm{GeV}^{2}$, will be presented.
\end{abstract}

Inelastic inclusive electromagnetic scattering can be described in term of a hadronic tensor for the target and a leptonic one for the beam. Symmetries reduce the hadronic tensor $W_{\mu \nu}^{A}$ to a combination of two unpolarized structure functions, $W_{1}$ and $W_{2}$, and two spin-dependent ones, $G_{1}$ and $G_{2}$, which are functions of the energy transfer $v$ and the four-momentum transfer $Q^{2}=-q^{2}$ :

$$
W_{\mu \nu}^{A}=2 \epsilon_{\mu \nu \lambda \sigma} q^{\lambda}\left\{M^{2} S^{\sigma} G_{1}\left(\nu, Q^{2}\right)+\left[M \nu S^{\sigma}-p^{\sigma} S \cdot q\right] G_{2}\left(\nu, Q^{2}\right)\right\} .
$$

The four structure functions, $G_{1}, G_{2}, W_{1}$ and $W_{2}$, contain all the information on nucleon structure that can be extracted from inclusive EM scattering. In the deep inelastic scattering (DIS) regime at high energy the structure functions scale, up to log corrections, as functions of only the Bjorken scaling variable $x=Q^{2} /(2 M v)$, with $M$ being the nucleon mass. The scaling spin dependent functions are

$$
\lim _{Q^{2}, v \rightarrow \infty}(M v) M G_{1}\left(v, Q^{2}\right)=g_{1}(x), \lim _{Q^{2}, v \rightarrow \infty}(M v) v G_{2}\left(v, Q^{2}\right)=g_{2}(x) .
$$

The longitudinal spin structure function (SSF) $g_{1}(x)$ can be described in terms of quark helicity distributions of each flavor, $g_{1}(x)=(1 / 2) \sum e_{q}^{2}\left(q_{q}^{\uparrow}(x)-q_{q}^{\downarrow}(x)\right), q=u, d, s, \ldots g_{2}$ has no equivalent form.

The hadronic tensor is also related, by the optical theorem, to the forward virtual Compton amplitude. Hence, the SSF's are related to the virtual photon absorption cross sections, $\sigma_{1 / 2}^{T}, \sigma_{3 / 2}^{T}$, for photon helicities $+1,-1$, and the transverse-longitudinal interference $\sigma_{1 / 2}^{T L}$. The spin asymmetries (SA)

\footnotetext{
a e-mail: or@virginia.edu
}

This is an Open Access article distributed under the terms of the Creative Commons Attribution License 4.0, which permits unrestricted use, distribution, and reproduction in any medium, provided the original work is properly cited. 
$A_{1}$ and $A_{2}$ are constructed from these cross sections and the unpolarized scaling $F_{1}(x)=M W_{1}\left(\nu, Q^{2}\right)$ :

$$
A_{1}=\frac{\sigma_{1 / 2}^{T}-\sigma_{3 / 2}^{T}}{\sigma_{1 / 2}^{T}+\sigma_{3 / 2}^{T}}=\frac{1}{F_{1}}\left(g_{1}-\gamma^{2} g_{2}\right), \quad A_{2}=\frac{\sigma^{T L}}{2 \sigma^{T}}=\frac{\gamma}{F_{1}}\left(g_{1}+g_{2}\right)=\frac{\gamma}{F_{1}} g_{T},
$$

where $2 \sigma^{T}=\sigma_{3 / 2}^{T}+\sigma_{1 / 2}^{T}, \gamma^{2}=(2 x M)^{2} / Q^{2}$, and $g_{T}$ is the transverse polarized structure function. Operators of both twist-2 (the "handbag" Feynman diagram) and twist-3 (quark-gluon correlations beyond the usual logarithmic or $\alpha_{S}$ corrections) contribute in leading order to the Compton amplitude for transverse polarized scattering. $g_{2}$ dominates the cross section difference for opposite beam helicities with orthogonal beam and target spins $\Delta \sigma_{\perp}=4 \alpha^{2} E^{\prime 2} \sin \theta \cos \phi\left(M G_{1}+2 E G_{2}\right) /\left(E Q^{2}\right)$. This unique feature of transverse polarized scattering allows access to sub-leading, twist-3 processes in a direct measurement [1], opening a window on the confinement of quarks and gluons inside nucleons.

In addition to probing twist-3 processes, $g_{2}$ is needed for applying higher twist corrections to the moments of $g_{1}$. The twist expansion of $g_{1}$ involves the $d_{2}$ matrix element, which is obtained from the third moments of $g_{2}$ and $g_{1}$. The predictions of lattice QCD, QCD sum rules, and quark models, can also be tested using the moments. At low $Q^{2}$, the polarizabilities of the color fields can be probed with $d_{2}$ (with the aid of the twist- $4 f_{2}$ matrix element). For the color magnetic field one has $\chi_{B}=\left(4 d_{2}+f_{2}\right) / 3$ and for the electric field $\chi_{E}=\left(4 d_{2}-2 f_{2}\right) / 3$. The 3rd moment is related to the color Lorentz force on transverse polarized quark [2], with the sign of $d_{2}$ related to the sign of the transverse deformation of the nucleon Finally, $g_{2}$ contains a chiral odd twist- 2 contribution, related to the quarks transverse spin (quark mass dependent term), which can be used to test quark masses (covariant parton models).

In terms of quark distributions $g_{T}(x)=(1 / 2) \sum e_{q}^{2} g_{T}^{q}(x)$. The $g_{T}^{q}(x)$ can be decomposed as [3]

$$
g_{T}^{q}(x)=\int d^{2} k_{t} \frac{\vec{k}_{t}^{2}}{2 M} \frac{g_{1 T}^{q}\left(x, \vec{k}_{t}^{2}\right)}{x}+\frac{m}{M} h_{1}^{q}(x)+\tilde{g}_{T}^{q}(x) .
$$

The first term is the twist- 3 chiral-even transverse momentum dependent distribution (TMD) $g_{1 T}^{q}\left(x, \vec{k}_{t}^{2}\right)$ where $k_{t}$ is the (unobservable) quark transverse momentum. The twist- 2 chiral-odd transversity TMD $h_{1}(x)$ is suppressed by the quark mass $m$. The last term, $\tilde{g}_{T}(x)$, represents twist-3 quark-gluon interactions. The twist-2 piece (Wandzura-Wilczek) of $g_{2}=g_{2}^{W W}+\bar{g}_{2}$

$$
g_{2}^{W W}\left(x, Q^{2}\right)=-g_{1}\left(x, Q^{2}\right)+\int_{x}^{1} \frac{d y}{y} g_{1}\left(y, Q^{2}\right),
$$

can be used to isolate twist-3 contributions from $q g$ interactions and from $g_{1 T}$. For each flavor we have

$$
g_{T}(x)=\int_{x}^{1} d y \frac{g_{1}(y)}{y}+\frac{m}{M}\left[\frac{h_{1}(x)}{x}-\int_{x}^{1} \frac{d y}{y} \frac{h_{1}(y)}{y}\right]+\tilde{g}_{T}(x)-\int_{x}^{1} \frac{d y}{y}\left(\tilde{g}_{T}(y)-\hat{g}_{T}(y)\right) .
$$

The twist-3 term $\hat{g}_{T}$ may not vanish in the Lorentz invariant relation [4] $g_{2}(x)=d g_{1 T}^{(1)}(x) / d x+\hat{g}_{T}(x)$.

The Spin Asymmetries of the Nucleon Experiment - SANE $^{1}$ was done at the Hall C facility of the Thomas Jefferson National Accelerator Facility - JLab, by the SANE collaboration[5]. The proton spin structure was measured in the region of $0.3 \leq x \leq 0.8$, for $2.5 \mathrm{GeV}^{2} \leq Q^{2} \leq 6.5 \mathrm{GeV}^{2}$, filling a major gap in the data on the transverse spin asymmetry. The experiment is documented on line [6].

The CEBAF accelerator delivered polarized electrons of 5.89 and $4.72 \mathrm{GeV}$, with average polarization $\left\langle P_{b}\right\rangle \simeq .73$, which scattered off polarized protons in solid ammonia $\left({ }^{14} \mathrm{NH}_{3}\right)$. Average inbeam proton polarizations $P_{t} \simeq .70$, for proton luminosity of $\simeq 10^{35} \mathrm{~Hz} \mathrm{~cm}^{-2}$, were attained by dynamic nuclear polarization with microwave pumping at $5 \mathrm{~T}$ and $1 \mathrm{~K}$. The $\mathrm{U}$. of Virginia polarized target was previously used at SLAC's and JLab in studies of the nucleon spin and the neutron charge form factor.

\footnotetext{
1 TJNAF E07-003, Spokespersons: O. Rondon (UVA), contact, Z-E. Meziani (Temple), S. Choi (Seoul), M. Jones (TJNAF).
} 
Table 1. Data collected by SANE.

\begin{tabular}{lccccc}
\hline Detector & Particle & Scattering & Beam Energy & Field Axis & Target \\
\hline BETA & $e, \pi^{0}$ & Inclusive Inelastic & $5.9,4.7 \mathrm{GeV}$ & $180^{\circ}, 80^{\circ}$ & $\mathrm{NH}_{3}$ \\
HMS & $e$ & Inclusive Inelastic & $5.9,4.7 \mathrm{GeV}$ & $180^{\circ}, 80^{\circ}$ & $\mathrm{NH}_{3}, \mathrm{C}, \mathrm{He}$ \\
& $e$ & Inclusive Elastic & $5.9 \mathrm{GeV}$ & $80^{\circ}$ & $\mathrm{NH}_{3}$ \\
BETA + HMS & $e-p$ & Coincidence Elastic & $5.9 \mathrm{GeV}$ & $80^{\circ}$ & $\mathrm{NH}_{3}$ \\
\hline
\end{tabular}
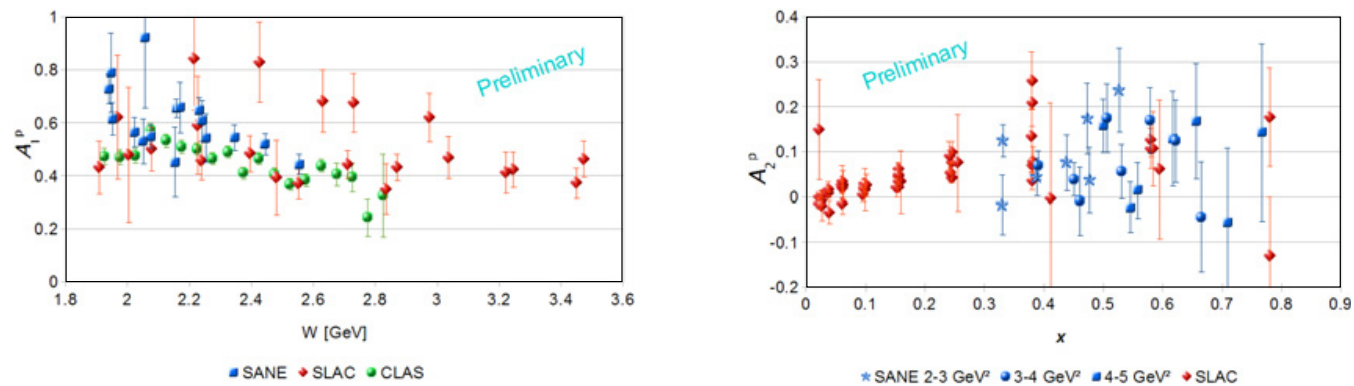

Figure 1. LEFT: SANE's preliminary 2012 results for $A_{1}^{p}$ plotted as a function of $W$ (squares). Results from SLAC $[8,9]$ (diamonds) and from CLAS [10] (circles) are also shown (CLAS results of different $Q^{2}$ values at the same $W$ bin have been merged). RIGHT: SANE's preliminary 2012 results for $A_{2}^{p}$ plotted as a function of Bjorken $x$ for three ranges of $Q^{2}$ (stars, circles and squares). Results from SLAC are shown as diamonds.

The scattered electrons in DIS kinematics were detected in the Big Electron Telescope Array (BETA), a novel large solid angle non-magnetic detector consisting of four subsystems: a 1792-elements lead glass calorimeter (BigCal), which was built for $G_{E}^{p} / G_{M}^{p}$ (E04-108/E04-019); a gas Cherenkov with a $5.9 \mathrm{GeV} / \mathrm{c}$ pion momentum threshold, built by Temple University; a 26-elements Lucite Cherenkov hodoscope built by North Carolina A\&T State U.; and a forward tracking hodoscope built by Norfolk State U. and U. of Regina. BETA's solid angle is 194 msr, after cuts.

Inclusive electron data at elastic and nucleon resonances kinematics were collected with the High Momentum Spectrometer - HMS. Elastic electron-proton coincidence data were also taken, with protons being detected in the HMS and electrons in BETA. A summary is given in Table 1.

Double spin asymmetries were measured for the anti-parallel $\left(180^{\circ}\right)$ and near-perpendicular $\left(80^{\circ}\right)$ orientations of the target field relative to the beam $\left(90^{\circ}\right.$ is not accessible). Asymmetries $\epsilon$ are formed from the difference over the sum of beam charge normalized and live time corrected yields $N^{+}, N^{-}$, for each beam helicity. The measured asymmetries for each orientation are $A_{m}=\epsilon /\left(f P_{b} P_{t}\right)$, where $f$ is the fraction of events scattered off polarized protons. The physics asymmetries $A\left(80^{\circ}\right), A\left(180^{\circ}\right)$ include corrections for the elastic radiative tail, internal and external radiative effects and elastic asymmetry, and for the background of $e^{+}, e^{-}$pairs resulting from $\pi^{0}$ Dalitz and $\gamma$ decays. The $A\left(80^{\circ}\right)$ and $A\left(180^{\circ}\right)$ asymmetries have been combined to obtain $A_{1}$ and $A_{2}$ with the aid of the unpolarized structure function $R$ [7]. The SSF's $g_{1}$ and $g_{2}$ are then obtained from $A_{1}$ and $A_{2}$ and $F_{1}$ [7].

Highlights of some preliminary results are shown in Figs. 1 and 2. $A_{1}^{p}(W)$ along with SLAC and CLAS data are shown on the left panel of Fig. 1. The corresponding results for $A_{2}$ are displayed on the right panel. $A_{2}$ is clearly non-zero. SANE error bars are total errors. The transverse SSF $g_{T}$ measures the component of the nucleon spin normal to the virtual photon. It best summarizes the effects of the power corrections to QCD scaling that represent the long range interactions (higher twists) between partons, beyond asymptotic freedom. A non-zero result for $g_{T}$ is a signature for HT. SANE's preliminary $g_{T}$ is displayed on the left panel of Fig. 2. The mean $\left\langle g_{T}(x>0.3)\right\rangle=0.023 \pm 0.006$ is about four sigmas greater than zero, a clear signature of HT. Quark-gluon correlations are best quantified by using the operator product expansion to connect twist-3 matrix elements with the third moments of $\bar{g}_{2}$. Nachtmann 

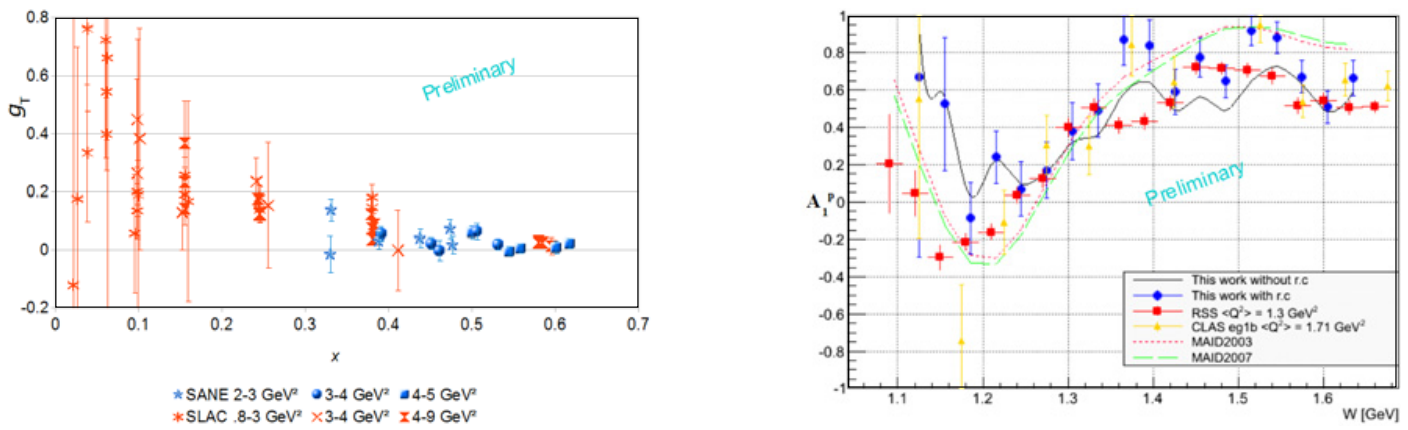

Figure 2. Left: transverse SSF $g_{T}^{p}$ for three ranges of $Q^{2}$. SANE's preliminary results are as labeled (blue symbols). SLAC's results in red. Right: SANE preliminary results for $A_{1}$ at $\sim 1.8 \mathrm{GeV}^{2}$ (blue circles) along with the $R S S$ $A_{1}[11]$ at $Q^{2}=1.3 \mathrm{GeV}^{2}$ (red squares) and the CLAS eg1b data at $1.8 \mathrm{GeV}^{2}$ (yellow triangles).

and CN moments of the SSF's will be calculated to extract the twist-2 matrix element $a_{0}, d_{2}$ and, possibly, $f_{2}$, after subtracting the twist- 2 target mass correction $a_{2}$.

The HMS coincidence and single arm elastic data have been used to determine preliminary values for the $G_{E} / G_{M}\left(Q^{2}\right)$ ratio from the beam-target asymmetry $G_{E} / G_{M}\left(2.1 \mathrm{GeV}^{2}\right)=.6 \pm .2$. The inelastic HMS data extend the kinematic range of the $R S S$ experiment at $Q^{2} 1.3 \mathrm{GeV}^{2}$ to $\sim 2.3 \mathrm{GeV}$. Data were also taken at $W$ around $1350 \mathrm{MeV}$ for $Q^{2} \simeq 1.8 \mathrm{GeV}^{2}$ where a resonance observed in the $R S S$ data has attracted interest from theoreticians [12]. A sample of preliminary results presented at DIS 2013 is shown on the right panel of Fig. 2, along with the $R S S A_{1}$, and Hall $\mathrm{B}$ eg $1 \mathrm{~b}$ data at $1.8 \mathrm{GeV}^{2}$.

This work was supported by DOE contract DE-FG02-96ER40950 and the Institute for Nuclear and Particle Physics of the University of Virginia.

\section{References}

[1] R.L. Jaffe, Comments Nucl. Part. Phys. 19, 239 (1990); arXiv: hep-ph/9602236

[2] M. Burkardt, AIP Conf. Proc. 1155, 26 (2009) [arXiv : 0905.4079 [hep-ph]]

[3] R.D. Tangerman and P. J. Mulders, hep-ph/9408305

[4] A. Accardi, A. Bacchetta, W. Melnitchouk and M. Schlegel, JHEP 0911, 093 (2009)

[5] https://hallcweb.jlab.org/experiments/sane/wiki/index.php/Image: SANE_collab.pdf

[6] http://hallcweb.jlab.org/experiments/sane/sane/

[7] M.E. Christy and P.E. Bosted, Phys. Rev. C 81, 055213 (2010) [arXiv : 0712.3731 [hep-ph]]

[8] SLAC E143, K. Abe et al., Phys. Rev. D 58, 112003 (1998)

[9] P.L. Anthony et al. [E155 Collaboration], Phys. Lett. B458, 529 (1999); P.L. Anthony et al. [E155 Collaboration], Phys. Lett. B 493, 19 (2000) P.L. Anthony et al. [E155 Collaboration], Phys. Lett. B553, 18 (2003)

[10] K.V. Dharmawardane et al. [CLAS Collaboration], Phys. Lett. B 641, 11 (2006)

[11] F.R. Wesselmann et al. [RSS Collaboration], Phys. Rev. Lett. 98, 132003 (2007)

[12] D. Drechsel, AIP Conf. Proc. 1155, 3 (2009); I. Strakovsky, private communication 